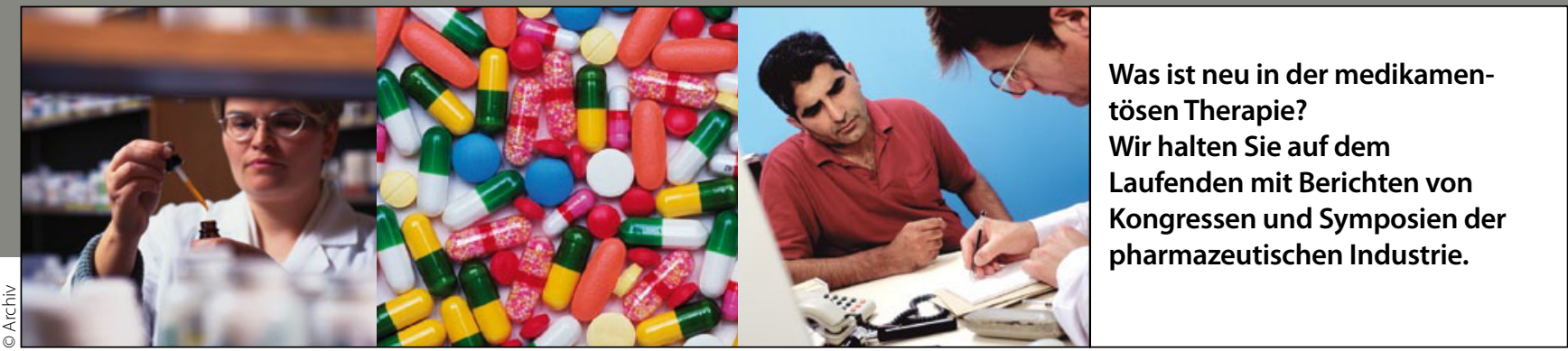

\title{
Meistens keine Indikation für Antibiotika
}

- In den Wintermonaten haben bis zu zwei Drittel der Patienten in allgemeinmedizinischen Praxen Atemwegsinfektionen. Meist sind die Erkrankungen viral bedingt, und nur bei Hinweisen auf eine bakterielle Genese ist eine Antibiotikatherapie sinnvoll. Das gilt besonders für die akute Bronchitis, so Prof. Adrian Gillissen vom Klinikum Kassel.

Lassen sich bei Verdacht Zeichen einer Pneumonie ausschließen und fehlen auch Hinweise auf eine bakterielle Infektion wie Fieber oder grün-gelbliches Sputum, dann werden bei akuter Bronchitis vor allem Mukopharmaka wie Ambroxol und Acetylcystein (ACC) empfohlen, die das Abhusten erleichtern sollen. Harte Studiendaten für ihren Einsatz fehlen jedoch, räumt Gillissen ein.

\section{Myrtol ebenso wirksam wie ein Antibiotikum}

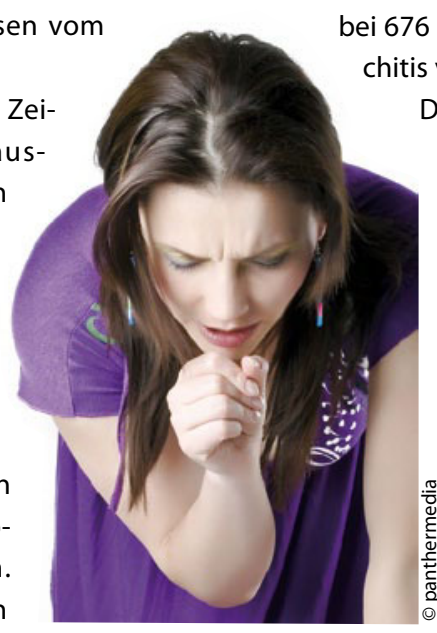

Myrtol erleichtert das Abhusten des Schleims.
Wegen ihres erweiterten Wirkspektrums bevorzugt der Internist und Pneumologe bei akuter Bronchitis Phytopharmaka wie GeloMyrtol $^{\circledR}$ forte (Myrtol standardisiert). "Das sind eigentlich ideale Medikamente, weil sie in vieler Hinsicht wirken", betonte Gillissen. So wurde für das pflanzliche Arzneimittel in Studien außer einer sekretolytischen und antientzündlichen Wirkung auch ein antimikrobieller Effekt belegt, und zwar gegen besonders häufige Atemwegskeime wie Pneumokokken und $\mathrm{H}$. influ- enzae. Auch fördert das Medikament die mukoziliäre Clearance und damit die Selbstreinigung der Bronchien.

In einer randomisierten Doppelblindstudie wurden die Wirksamkeit von Myrtol standardisiert, dem Antibiotikum Cefuro-

xim sowie von Ambroxol und Placebo bei 676 Patienten mit akuter Bronchitis verglichen.

Das Ergebnis: Nach 14 Tagen lagen die Responderraten bei allen aktiv Behandelten über $90 \%$, im Vergleich zu $85 \%$ unter Placebo. Keine Unterschiede bei den Therapieergebnissen gab es zwischen Myrtol standardisiert und dem Antibiotikum. Die Auskultationsbefunde nach 14 Tagen @ sprachen für eine ebenbürtige Wirksamkeit von Myrtol standardisiert und Cefuroxim, beide Substanzen waren dabei Ambroxol und Placebo deutlich überlegen. 79\% der Ärzte beurteilten die Wirksamkeit von Myrtol standardisiert mit "gut" oder "sehr gut", im Vergleich zu 74\% (Cefuroxim), 66\% (Ambroxol) und 42\% (Placebo).

\section{Antibiotikum-Rezept \\ für den Bedarfsfall}

In der Annahme, die Patienten erwarteten es nicht anders, verschreiben Ärzte manchmal wider besseres Wissen Antibiotika. Die vorauseilende Wunscherfüllung ist aber meist überflüssig, wie eine Umfrage von Robert Koch-Institut und Charité ergeben hat (Euro Surveillance online). In der Studie wurden 1076 Personen im Alter von 15 bis 78 Jahren über ihre Einstellungen zu Antibiotika befragt. Nur gut jeder Zehnte gab an, dass er erwarte, bei einer Erkältung ein Antibiotikum zu bekommen. Und von diesen würden wiederum mehr als drei Viertel die ärztliche Entscheidung gegen ein solches Medikament akzeptieren.

Kollegen haben also keine Nachteile zu befürchten, wenn sie bei Erkältungen mit Antibiotika zurückhaltend sind. Im Zweifelsfall hat es sich bewährt, den Patienten ein Rezept für ein Antibiotikum auszustellen, das sie nur bei Bedarf einlösen.

- Wolfgang Geissel

Quelle: Gespräch mit Prof. A. Gillissen;

Arznm.-Forsch./Drug Res 50 (8); 2000: 700)

\section{Wenn der Husten persistiert}

\section{Wann Antitussiva, wann Antibiotika?}

Den bronchialen Infekt kann ein postinfektiöser Husten um mehrere Wochen überdauern. Wird dabei die Lebensqualität etwa durch gestörten Schlaf wesentlich beeinflusst, empfiehlt die Deutsche Gesellschaft für Pneumologie eine bis zu 14 Tage dauernde Therapie mit zentral wirksamen Antitussiva wie Codein.

Antibiotika sind zum Beispiel bei bakteriellen Superinfektionen einer akuten Bronchitis indiziert. Kennzeichen können verschlechterte Symptome bei einer meist verschleppten Erkrankung sein, ebenso Fieber, eitriges Sputum oder erste Symptome einer Pneumonie. 